Volume 9. No.12, December 2021

International Journal of Emerging Trends in Engineering Research

Available Online at http://www.warse.org/IJETER/static/pdf/file/ijeter039122021.pdf

https://doi.org/10.30534/ijeter/2021/039122021

\title{
Effect of Preliminary Deformation on Microstructure and Texture of Iron-Chromium Alloy Prepared by Severe Plastic Deformation
}

\author{
Muhammad Rifai $^{1}$, Mujamilah ${ }^{1}$, Hiroyuki Miyamoto ${ }^{2}$ \\ ${ }^{1}$ Center for Research and Technology of Nuclear Advanced Material, National Research and Innovation Agency, \\ Serpong, Tangerang Selatan, Banten, Indonesia \\ ${ }^{2}$ Department Science and Engineering, Doshisha University, Kyotanabe Campus, Kyoto, Japan
}

Received Date : November 06, 2021 Accepted Date : November 28, 2021 Published Date : December 07, 2021

\begin{abstract}
The effect of preliminary deformation on the microstructure and texture of iron-chromium alloy prepared by severe plastic deformation (SPD) has been investigated in grain refinement and inhomogeneity structure. Equal channel angular pressing (ECAP) is a well-known SPD process that uses a die channel with a sharp angle. The texture and misorientation map of ECAP processed material was observed electron backscattered diffraction (EBSD) analysis, providing information on structure evolution. The observation was done in the transverse plane from the middle to the sub-surface. The data logger also records the pressure of the ECAP process. The result showed that the sub-surface has a more deformed structure than the middle due to the die channel's sharp angle and shear direction. The texture exhibited a random orientation after the first pass ECAP process. The stacking fault energy and accumulation dislocation are also associated with this process. Several shear bands can be seen clearly, which is parallel to the shear direction. It concluded that the preliminary deformation by ECAP was effective to promote grain refinement due to their high equivalent strain.
\end{abstract}

Key words : Deformation, ECAP, SPD, Alloy

\section{INTRODUCTION}

The severe plastic deformation (SPD) technique is well-known to promote grain refinement by accumulation dislocation inside grain and grain boundary [1-3]. Equal channel angular pressing (ECAP) is an SPD technique that uses a billet as a rod or bar that does not change the billet dimension [4-6]. ECAP was used in this study due to their highest equivalent strain among other techniques such as high-pressure torsion (HPT) [7-9] and accumulative roll bonding (ARB) [10-12]. ECAP could also introduce a significant strain and promote ultrafine-grained (UFG) structure efficiently by reducing the grain size.
The previous report did not discuss the effect of preliminary deformation during the ECAP process on the microstructure and texture of pure crystal structures like BCC in detail in terms of microstructure and texture behaviour [13-16]. The chromium alloy is used to identify and confirm the characteristic of the BCC metallic material. The ECAP process has several parameter processes associated with microstructure evolution during the deformation process, such as pressure speed, route of pass number, and angle of die channel $[17,18]$. It is essential to investigate more detail on this preliminary deformation during the ECAP process due to a unique material characteristic of pure metal or crystal structure. The size and die angle are also associated with the backpressure of the material due to the high deformation level during the ECAP process $[19,20]$. This back pressure of one pass at the ECAP process may contribute to grain fragmentation at a specific angle due to the die channel. Lamellar and band structure can be easily seen after one pass, and it may influence the texture and structure of the material.

The previous studies confirmed that the texture analysis could be used to explain the share deformation behavior during the ECAP process [4-7]. Simple share deformation occurred at the ECAP process, which is perpendicular to the rolling plane. There is no publication that discusses the texture analysis on the preliminary deformation during ECAP in detail. The hypothesis said that the preliminary deformation might influence the texture of material, which can be seen in the pole figure by electron backscattered diffraction (EBSD) analysis. The EBSD analysis provides the orientation grain and texture eventually on the material. This study aims to investigate the effect of the preliminary deformation of iron-chromium alloy on microstructure evolution and texture by EBSD analysis. 


\section{EXPERIMENTAL PROCEDURE}

Iron chromium alloy was prepared by $8 \mathrm{~mm} \times 8 \mathrm{~mm}$ and 100 $\mathrm{mm}$ length of billet. The billets were heat-treated at $823 \mathrm{~K}$ for 2 hours. The ECAP process was carried out using the 90-degree inner angle of the die channel and a $423 \mathrm{~K}$ temperature process. The initial grain of heat-treated material is around $450 \mu \mathrm{m}$. The microstructure observation was carried out at the transverse plane and perpendicular to the rolling plane of the die channel. The billet was inserted into the die channel and recorded the backpressure by the data logger. The $500 \mathrm{kN}$ Shimadzu Autograph was used to carry out the ECAP process. Vicker Hardness Shimadzu measured the hardness. The ECAP processed sample was grounded and polished to observe by JEOL FE-SEM 7001 and analyze by EBSD for orientation map and texture. The structure and texture were observed from the middle to the sub-surface of the billet, with a range of $100 \mu \mathrm{m}$ for each point. The INCA software was used to provide the orientation map at the transverse, normal and rolling plane at the EBSD system, as seen in Figure 1. The right image explain about the sample shear direction during ECAP process which use 90-degree inner channel angle, and the left image shows the shear deformation during ECAP.

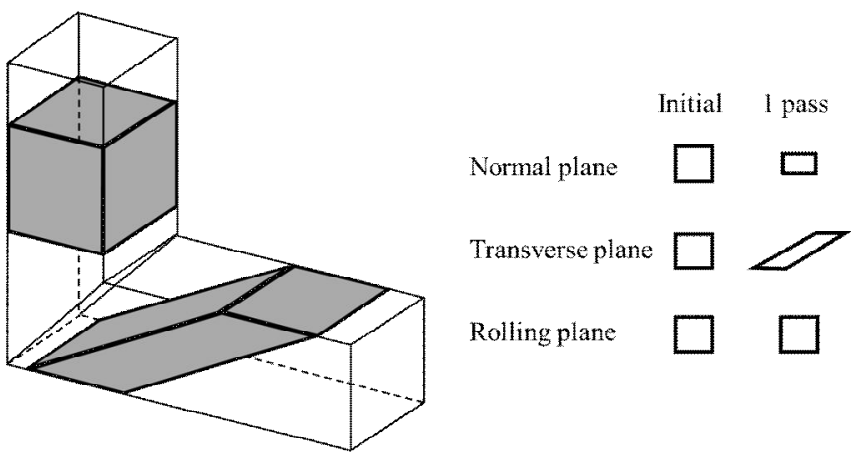

Figure 1. The shear formation in the first ECAP pass at the normal, transverse and rolling plane

\section{RESULT AND DISCUSSION}

The orientation image map of the heat-treated sample can be seen in Figure 2. The misorientation map (Figure 2a) of the annealed sample showed larger grains with [111], [101] and [001] orientation plane.; and Figure $2 \mathrm{~b}$ shows the pole figure of the random orientation of [111], [101] and [001], which indicate the un-deformed structure. The EBSD analysis of the heat-treated sample exhibited equiaxed and large grain due to high annealing temperature, in which the elongated grain and initial deformation were removed from the sample. The texture before the ECAP process showed a random texture in a specific orientation, in which the grain is recrystallized. The high angle grain boundary was seen in the high contrast boundary line at pattern image by EBSD.

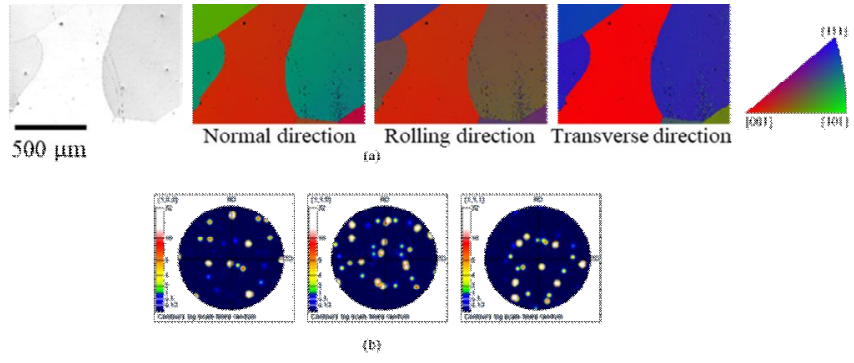

Figure 2. (a) the orientation map and (b) the texture appearance of the as-annealed sample.

Backpressure during the ECAP process can be seen in Figure 3 , in which the highest initial deformation occurred until 105 $\mathrm{kN}$ and suddenly decrease to $78 \mathrm{kN}$. This result was confirmed by increasing the Vickers Hardness from $125 \mathrm{Hv}$ to $225 \mathrm{Hv}$ after one pass. The heat-treated sample hardness was below the as-received sample as $150 \mathrm{Hv}$, which similar at $\mathrm{FeCrNi}$ alloy [21], and it happened because the subsequent recrystallization has occurred efficiently after heat treatment.

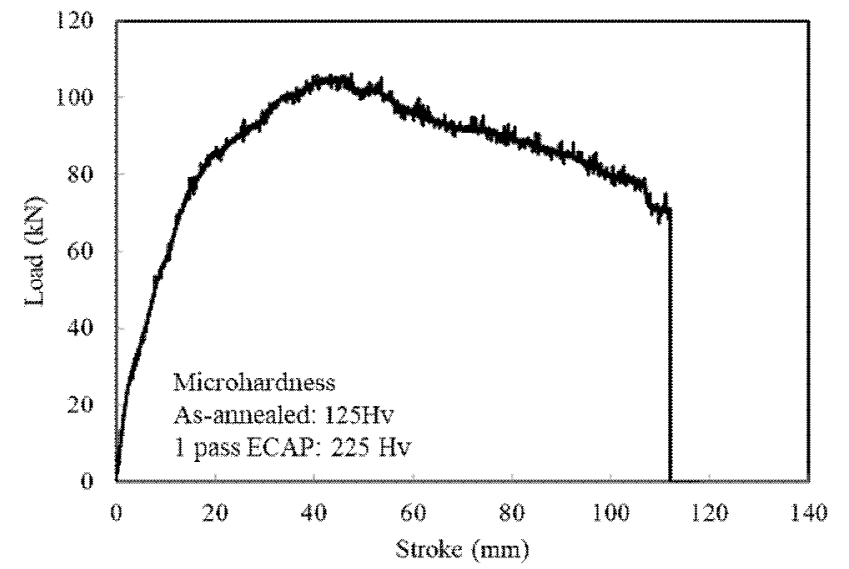

Figure 3. The ECAP pressure and microhardness result for the first-pass number on the iron-chromium alloy.

Figure 4 shows the misorientation map from the middle to sub-surface by defining the D/Do position. The shear band and lamellar structure were observed clearly in the middle of the billet. The grain fragmentation has occurred along the rolling plane with $26.2^{\circ}$ of shear angle. This study is carried out at a $423 \mathrm{~K}$ temperature due to hard metallic material. The ECAP process is efficient in low temperatures due to the nonappearance of dynamic recovery and recrystallization, which confirmed with other results [15,16]. Iron chromium alloy has medium stacking fault energy, which can use the discontinuous dynamic recrystallization for grain refinement by increasing the temperature process until $423 \mathrm{~K}$ [15]. The higher energy strain of iron-chromium can efficiently promote grain refinement discontinuous dynamic recrystallization. 


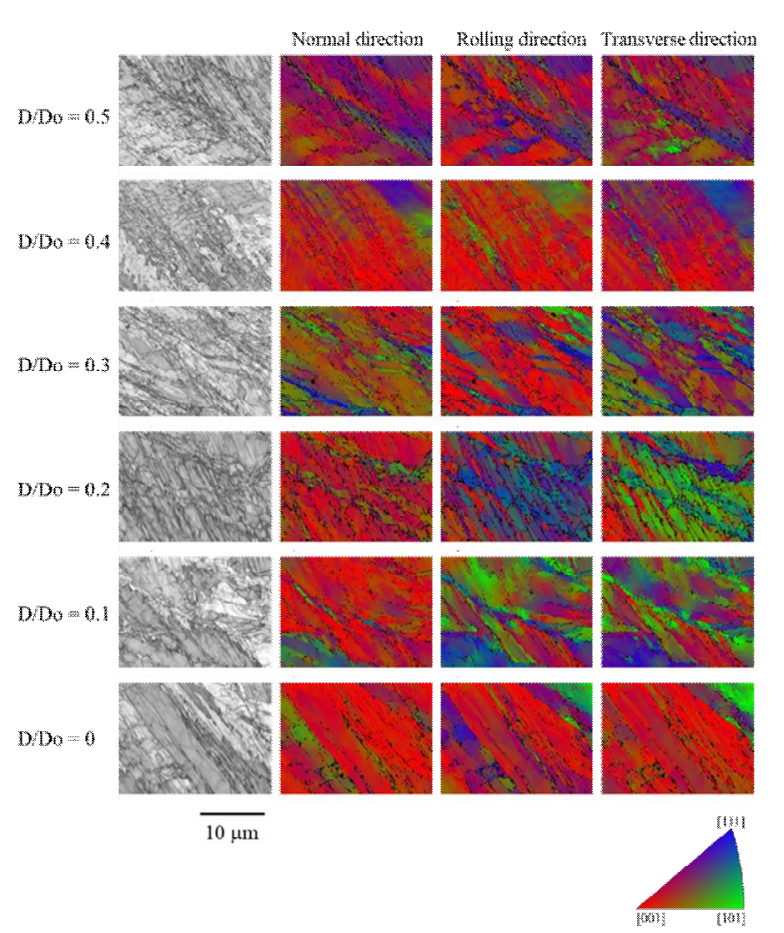

Figure 4. The orientation map of the first ECAP passes from the middle to sub-surface on the transverse plane by D/Do ratio.

Figure 5 shows the texture of one pass ECAP processed sample at several positions based on D/Do. It seems the sub-surface exhibited a random texture compared to other positions due to the higher deformation level at the sub-surface during the ECAP process [14,15]. The 110 plane showed the most random texture due to the BCC crystal structure. The sub-grain structure with 5-10 $\mu \mathrm{m}$ grain size has been seen after one pass in the middle billet, it confirmed that the grain refinement was effectively occurred during ECAP process.

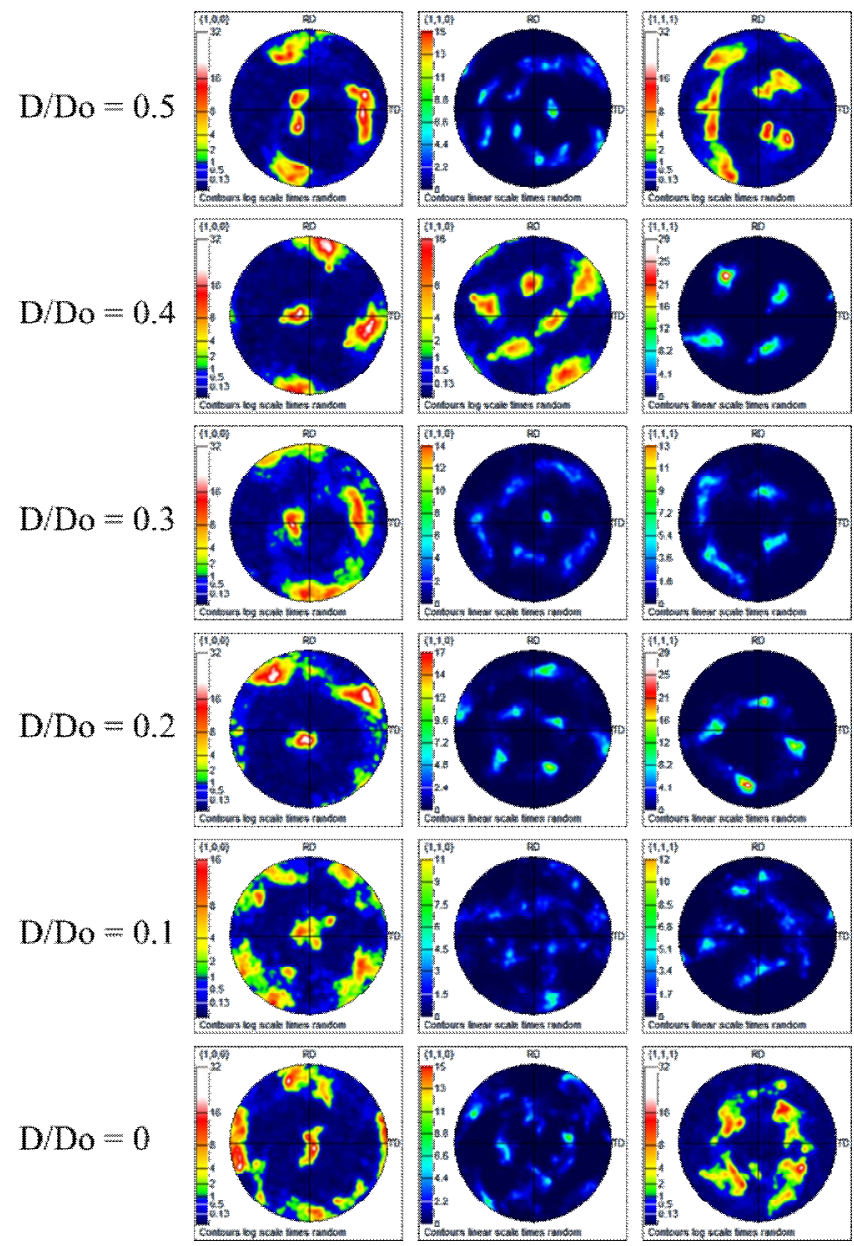

Figure 5. The texture (pole figure) of the first ECAP pass from the middle to sub-surface on the transverse plane by D/Do ratio.

\section{CONCLUSION}

The ECAP process was effectively promoted grain fragmentation and shear band at iron-chromium alloy, which can continue to grain refinement after one pass of ECAP. The measurement of the grain size was carried out by INCA software which installed at SEM/EBSD. The subsequent recrystallization was occurred efficiently at the heat-treated sample before processing by ECAP. As a result, there is an increment in hardness after one pass, which is associated with to backpressure result. The higher energy strain of iron-chromium can promote grain refinement efficiently due to the discontinuous dynamic recrystallization. The preliminary deformation on iron-chromium alloy as BCC metallic material can be a reference for next research on other crystal structures.

\section{REFERENCES}

[1] T. C. Lowe, and R. Z. Valiev. Investigations and applications of severe plastic deformation, Springer Science \& Business Media, 2012.

[2] G. Faraji, H. S. Kim, and H. T. Kashi. Severe plastic deformation: methods, processing and properties, 
Elsevier, 2018.

[3] B. Verlinden. Severe plastic deformation of metals, Metallurgical and Materials Engineering. 2018.

[4] S. Frint, M. Hockauf, P. Frint and M. F. X. Wagner. Scaling up Segal's principle of equal-channel angular pressing, Materials \& Design, vol. 97, pp. 502-511, 2016.

[5] R. A. Parshikov, A. I. Rudskoy, A. M. Zolotov and O. V. Tolochko. Technological problems of equal channel angular pressing, Rev. Adv. Mater. Sci, vol. 34, pp. 26-36, 2013.

[6] M. A. Agwa, M. N. Ali, and A. E. Al-Shorbagy. Optimum processing parameters for equal channel angular pressing, Mechanics of Materials, vol. 100, pp. 1-11, 2016.

[7] K. Edalati, and Z. Horita. A review on high-pressure torsion (HPT) from 1935 to 1988, Materials Science and Engineering: A, vol. 652, pp. 325-352, 2016.

[8] K. Edalati, Y. Hashiguchi, P. H. R. Pereira, Z. Horita and T. G. Langdon. Effect of temperature rise on microstructural evolution during high-pressure torsion, Materials Science and Engineering: A, vol. 714, pp. 167-171, 2018.

[9] F. A. Mohamed, and S. S. Dheda. On the minimum grain size obtainable by high-pressure torsion, Materials Science and Engineering: A, vol. 558, pp. 59-63, 2012.

[10]S. M. Ghalehbandi, M. Malaki and M. Gupta. Accumulative roll bonding-a review, Applied Sciences, vol. 9, no. 17, pp.3627, 2019.

[11] M. R. Toroghinejad, F. Ashrafizadeh, and R. Jamaati. On the use of accumulative roll bonding process to develop nanostructured aluminum alloy 5083. Materials Science and Engineering: A, vol. 561, pp.145-151, 2013.

[12] A. Fattah-Alhosseini, and O. Imantalab. Effect of accumulative roll bonding process on the electrochemical behavior of pure copper, Journal of alloys and compounds, vol. 632, pp. 48-52, 2015.

[13] M. Rifai, H. Miyamoto, and H. Fujiwara. Effects of strain energy and grain size on corrosion resistance of ultrafine grained $\mathrm{Fe}-20 \% \mathrm{Cr}$ steels with extremely low $\mathbf{C}$ and $\mathbf{N}$ fabricated by ECAP, International Journal of Corrosion, 2015.

[14] M. Rifai, H. Miyamoto, and H. Fujiwara. Effect of ECAP deformation route on the degree of anisotropy of microstructure of extremely low CN Fe-20mass\% Cr alloy, Metals, Vol. 4, No. 1, pp. 55-63, 2014.

[15]M. Rifai, M. Yuasa, and H. Miyamoto. Enhanced corrosion resistance of ultrafine-grained $\mathrm{Fe}-\mathrm{Cr}$ alloys with subcritical $\mathbf{C r}$ contents for passivity, Metals, vol. 8, no. 3, p.149, 2018.

[16] M. Rifai, H. Miyamoto. Effect of strain energy on the grain growth behaviour of ultrafine-grained iron-chromium alloy by equal channel angular pressing, Journal of Mechanical Engineering and Sciences, vol. 14, no. 3, pp.7049-7057, 2020.

[17] P. C. Gautam, and S. Biswas. On the possibility to reduce ECAP deformation temperature in magnesium: Deformation behaviour, dynamic recrystallization and mechanical properties, Materials Science and Engineering: A, vol. 812, p.141103, 2021.

[18] V. Bratov, and E. N. Borodin. Comparison of dislocation density based approaches for prediction of defect structure evolution in aluminium and copper processed by ECAP, Materials Science and Engineering: A, vol. 631, pp.10-17, 2015

[19]P. Minárik, M. Zemková, J. Veselý, J. Bohlen, M. Knapek, and R. Král. The effect of $\mathrm{Zr}$ on dynamic recrystallization during ECAP processing of Mg-Y-RE alloys, Materials Characterization, vol. 174, p.111033, 2021.

[20] N. Koptseva, Y. Efimova, M. Chukin, I. Yakovleva, A. Zhilyaev, and E. Korznikova. Kinetics of dynamic recrystallization in low-and medium carbon steels during warm ECA pressing, Materials Letters, vol. 285, p.128954.

[21] M. Dani, S. Mustofa, S. Parikin, T. Sudiro, B. Hermanto, D. R. Adhika, A. Insani, A. Dimyati, S. H. Syahbuddin, E. A. Basuki, and C. A. Huang. Effect of Spark Plasma Sintering (SPS) at Temperatures of $900 a n d 950$ o c for 5 Minutes on Microstructural Formation of Fe-25Ni-17Cr Austenitic Stainless Steel, International Journal of Emerging Trends in Engineering Research, vol. 8, no. 8, 2020. 